\title{
Persistent left superior vena cava discovered during pacemaker implantation in a paediatric patient
}

\author{
Damian Małecki ${ }^{1, \mathrm{~B}-\mathrm{E}}$, Piotr Wieniawski ${ }^{2, \mathrm{~A}-\mathrm{B}, \mathrm{E}}$, Bożena Werner ${ }^{2, \mathrm{~A}-\mathrm{B}}$, Grzegorz Opolski ${ }^{1, \mathrm{~A}, \mathrm{~F}}$, Marcin Grabowski ${ }^{1, \mathrm{~A}-\mathrm{B}, \mathrm{E}-\mathrm{F}}$ \\ A - Research concept and design, B - Collection and/or assembly of data, C - Data analysis and interpretation, \\ D - Writing the article, E - Critical revision of the article, F - Final approval of article \\ $11^{\text {st }}$ Department of Cardiology, Medical University of Warsaw \\ 2 Department of Paediatric Cardiology and General Paediatrics, Medical University of Warsaw
}

Address for correspondence:

Damian Małecki, $1^{\text {st }}$ Department of Cardiology, Medical University of Warsaw email: damian.malecki@student.wum.edu.pl

Piotr Wieniawski, Department of Paediatric Cardiology and General Paediatrics, Medical University of Warsaw email: piotr.wieniawski@spdsk.pl

Bożena Werner, Department of Paediatric Cardiology and General Paediatrics, Medical University of Warsaw email: bozena.werner@wum.edu.pl

Grzegorz Opolski, $1^{\text {st }}$ Department of Cardiology, Medical University of Warsaw email: grzegorz.opolski@wum.edu.pl

Marcin Grabowski, $1^{\text {st }}$ Department of Cardiology, Medical University of Warsaw email: marcin.grabowski@wum.edu.pl

Received: 2019-11-04

Revised:

Accepted: 2019-11-10

Final review: 2019-11-10

DOI: $10.24255 / \mathrm{hbj} / 114124$

\section{Key words:}

persistent left superior vena cava, pacemaker implantation, paediatric, cardiac stimulation, lead implantation complications

\section{Introduction}

A 12-year-old boy with a history of congenitally corrected transposition of the great arteries (ccTGA) underwent pacemaker (PM) implantation due to advanced conduction disorders (numerous episodes of 1st, 2nd and 3rd degree atrioventricular (AV) block). During the procedure, persistent left superior vena cava (PLSVC) was discovered. Although this finding required supplementary manoeuvring, the ventricular lead was successfully placed into the sub-pulmonary chamber and the procedure was completed without complications.

\section{The patient's profile overview}

The patient was a 12-year-old boy, with congenital heart anomaly (CHA) - ccTGA. In this defect heart ventricles are reversed - the left, systemic ventricle is morphologically the right ventricle, while the right, sub-pulmonary ventricle is morphologically left. The patient developed multiple arrhythmias - episodes of 1st, 2nd and 3rd degree AV block as well as self-limiting supraventricular tachycardia. The patient was qualified for pacemaker implantation during multi-specialist consultation. Pre-implantation diagnostic measures were taken - Holter ECG monitoring confirmed AV blocks, while transthoracic echocardiography (TTE) and magnetic resonance imaging (MRI) ruled out potential obstacles to the procedure.

\section{Procedure description}

The implantation procedure took place in the operating theatre, under general anaesthesia, and was performed by a paediatric cardiac surgeon and an electrophysiology cardiologist. An incision in the left subclavian e.g. region was made and the cephalic vein was found. Selective venography was done, and presence of the PLSVC was discovered (Fig. 1 \& 2), although such a finding was not described in any of the previous imaging 


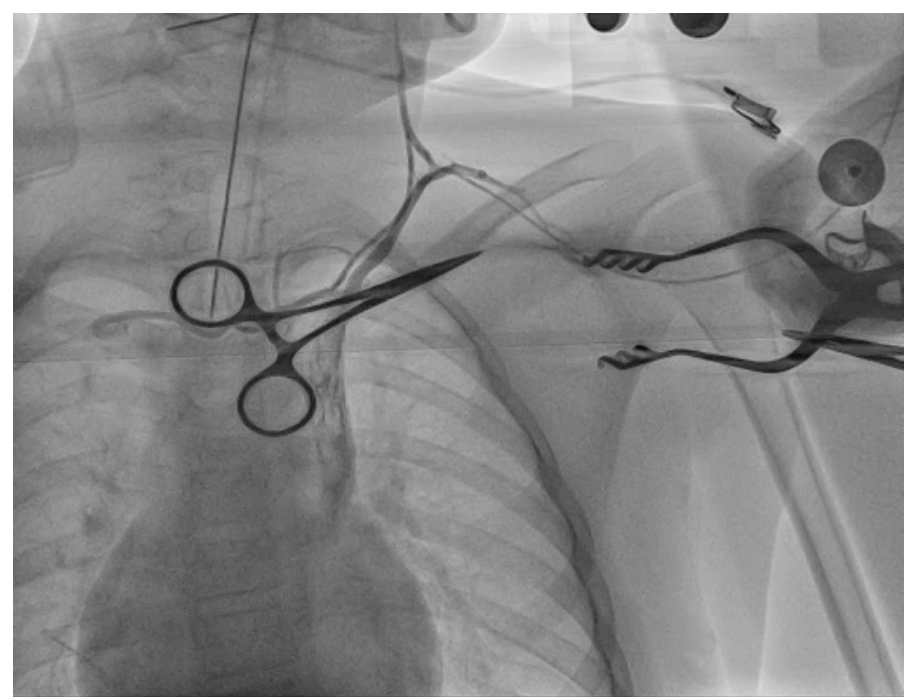

Figure 1.

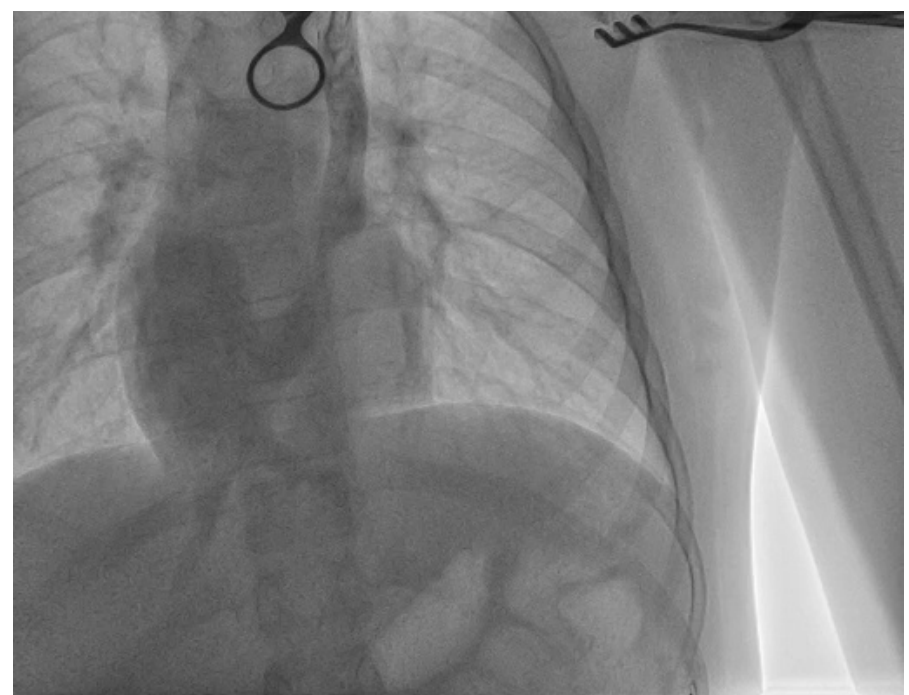

Figure 2 .

examinations. The stylet was introduced into the cephalic vein through venesection. It was later descended through the PLSVC and coronary sinus (CS) into the right atrium (RA) (Fig. 3). The lead was then slid down into the RA over the stylet.

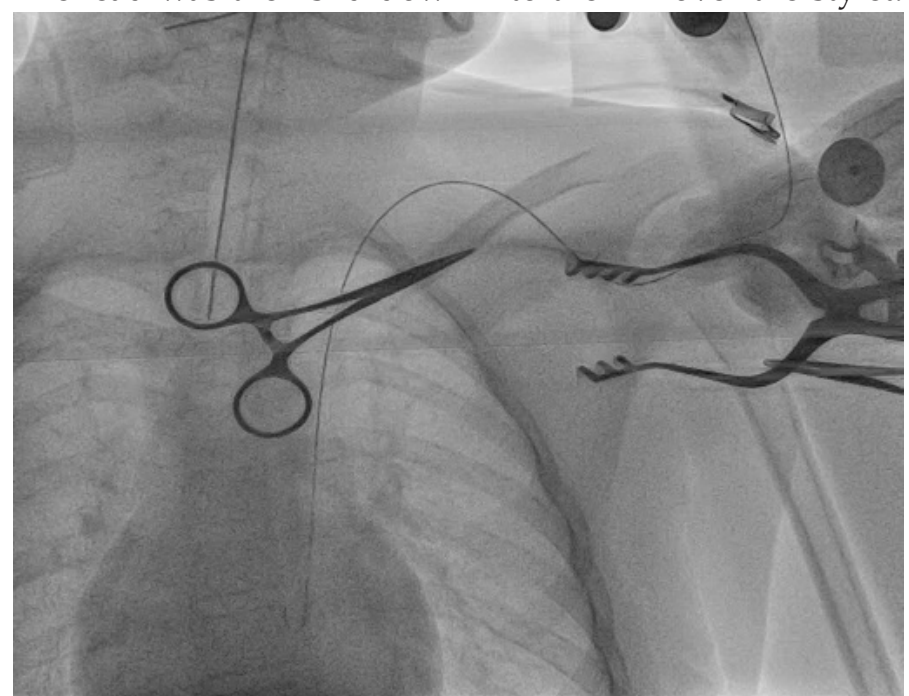

Due to non-standard anatomy of the heart with ccTGA and existence of PLSVC, multiple attempts to introduce the lead into the sub-pulmonary ventricle were made with pigtail and J-shape stylets. During one such unsuccessful attempt the lead retrogradely positioned itself in the right superior vena cava (Fig. 4). Finally, the lead was positioned in the apical region

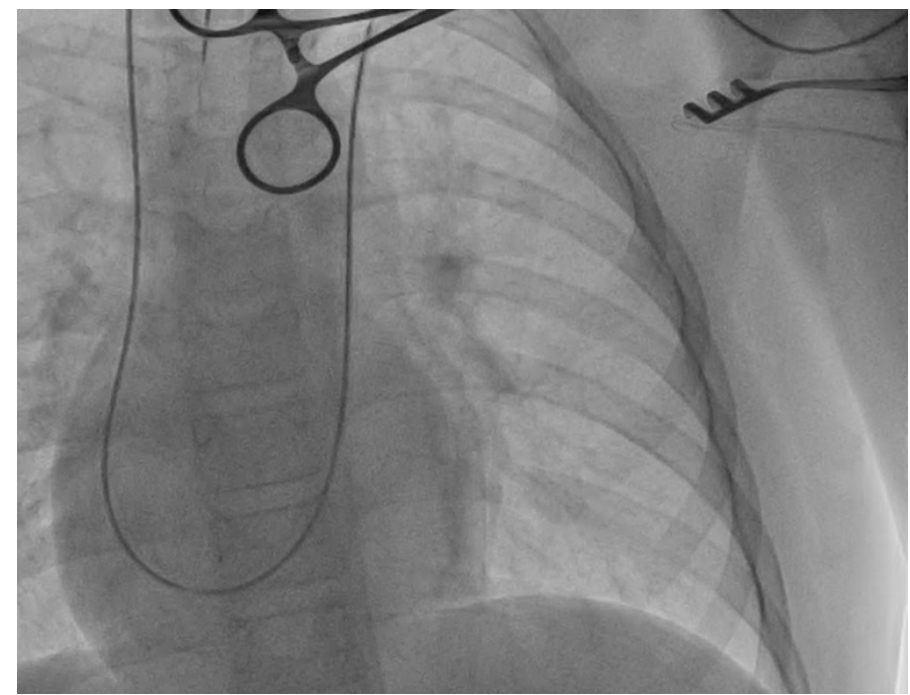

Figure 4.

of the sub-pulmonary ventricle, stimulation parameters were checked, and the lead position was secured (Fig. 5). The lead was connected to the pacemaker. The procedure was finished and periprocedural outcome was uneventful.

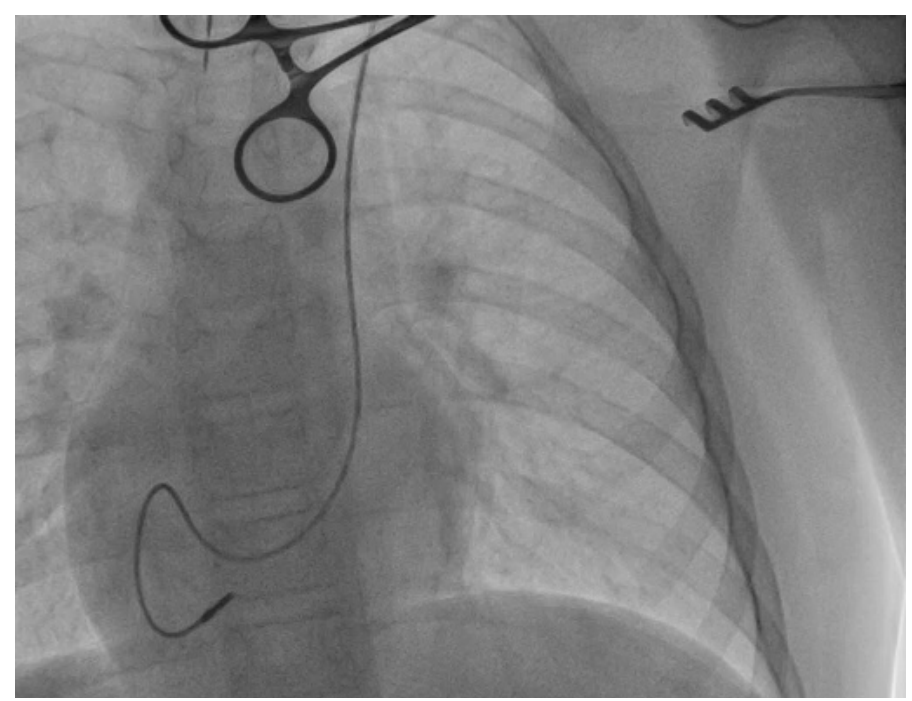

Figure 5 .

\section{Disscusion}

PLSVC is the most common venous anomaly, presenting in $0.3 \%-0.5 \%$ of patients in the general population ${ }^{[1-3]}$, but with much higher incidence in patients with $\mathrm{CHA}$, up to $11 \%{ }^{[4]}$. PLSVC is usually asymptomatic and has no haemodynamic significance but can create a challenge during PM implantation $^{[5,6]}$. Dilated coronary sinus and sharp angle of passing through the tricuspid valve require use of multiple shapes 
of stylets and additional modelling of this tool ${ }^{[6]}$. Although the mentioned methods apply to adult patients, they can be successfully implemented in a paediatric procedure.

Our patient was considered complicated in advance due to ccTGA. This anomaly causes the heart to be rotated and shifted to the right (Fig. 6), creating unusual angles of access to the ventricle. Intraprocedural discovery of the PLSVC added
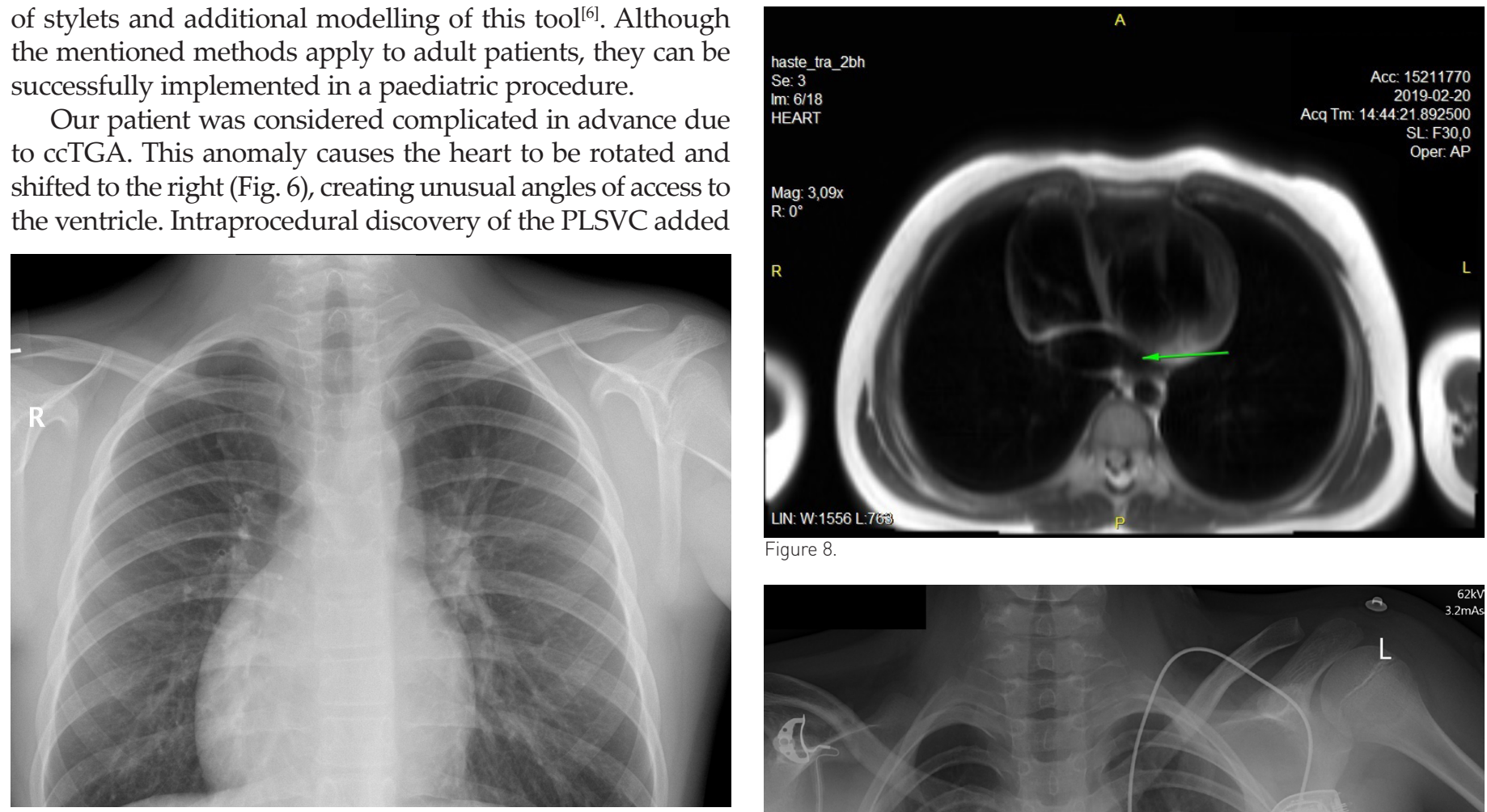

Figure 8.

Figure 6

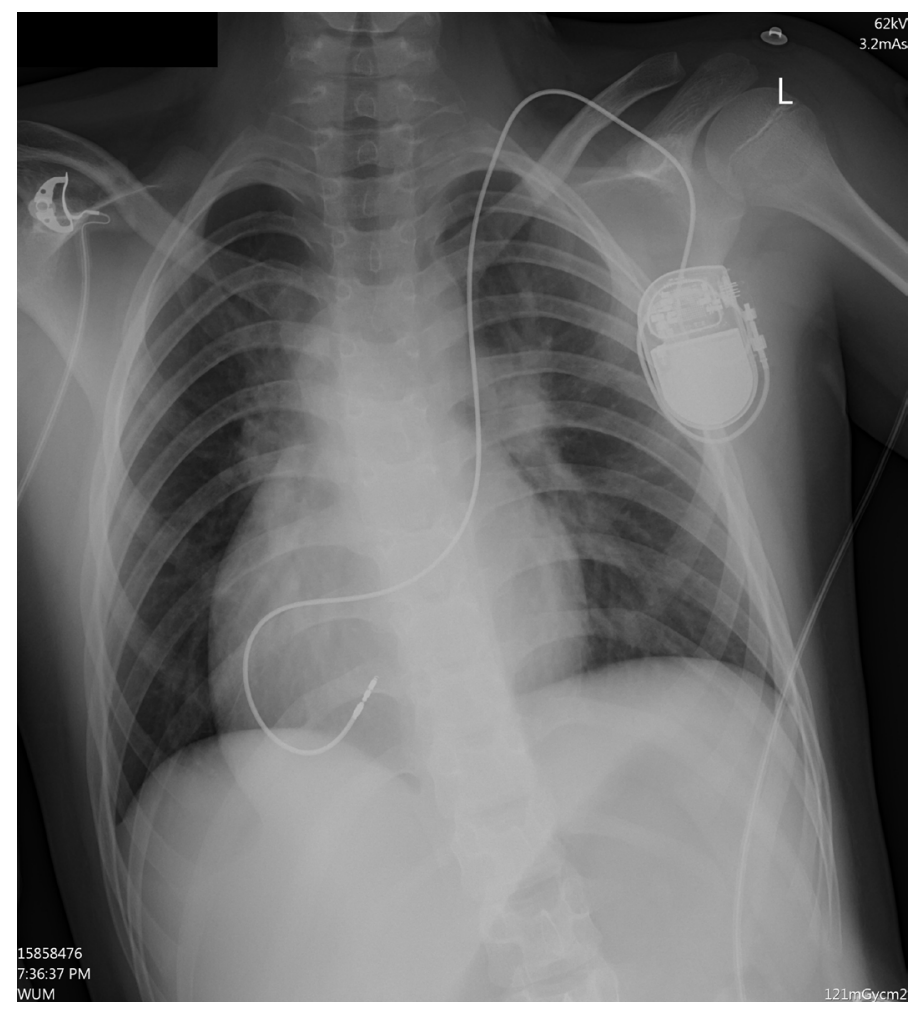

Figure 8 .

even more complexity to the procedure. It is unclear why the PLSVC was not noticed during the MRI examination. Later re-examination of the MRI sequence allowed selection of a structure that may be the PLSVC (located laterally to the left atrium) and dilated CS (Fig. 7 \& 8). ccTGA in combination

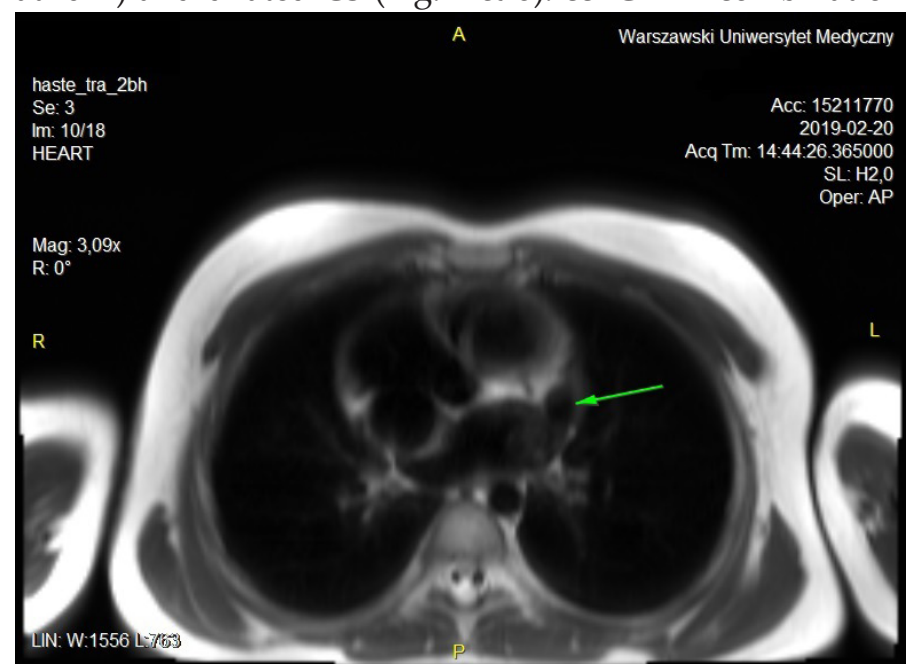

Figure 7.

be also suspected if a dilated coronary sinus is discovered in TTE. A contrast injection test during TTE is suggested for diagnosis of PLSVC ${ }^{[7]}$. If it is present, contrast injected into the left antecubital vein will be visible in the CS first and RA later.

\section{Conclusion}

with PLSVC required skilful lead manoeuvring, so that it could be placed in the sub-pulmonary ventricle. These anomalies significantly extended the duration of the procedure, which is typical for procedures on patients with PLSVC ${ }^{[5]}$.

PLSVC is usually diagnosed during invasive procedures, such as central venous catheterization, PM implantation or retrograde cardioplegia for cardiac surgery. Fluoroscopic or $X$-ray images reveal the catheter or PM lead to descend on the left side of the spine (Fig. 9), not crossing the spine and descending in the right superior vena cava field. PLSVC may

Combination of congenital heart anomaly and existence of PLSVC significantly prolonged the duration of the procedure and required excessive measures to achieve optimal lead location and pacing parameters. PLSVC may be suspected in patients with dilated CS and suggestive position of intravenous instruments, such as catheters or PM leads. A simple contrast echocardiographic test may be beneficial for diagnosis of PLSVC. 


\section{References}

1. Petrac D, Radeljica V, Pavlovica N, Manolaa S, Delic-Brkljacica D. Persistent Left Superior Vena Cava in Patients Undergoing Cardiac Device Implantation: Clinical and Long-Term Data. Cardiol Res 2013;4(2):64-67

2. Tak T, Crouch E, Drake GB. Persistent left superior vena cava: incidence, significance and clinical correlates. Int J Cardiol 2002;82:91-93

3. Perles Z, Nir A, Sagui G et al. Prevalence of Persistent Superior Vena Cava and Association With Congenital Heart Anomalies. Am J Cardiol 2013;112:1214-1218

4. Galindo A, Gutiérrez-Larraya F, Escribano D, Arbues J, Velasco JM, Gutiérrez-Larraya F. Clinical significance of persistent left superior vena cava diagnosed in fetal life. UltrasoundObstetGynecol 2007;30:152-161.

5. Li T, Xu Q, Liao H, Asvestas D, Letsas KP, Li Y. Transvenous dual-chamber pacemaker implantation in patients with persistent left superior vena cava. BMC Cardiovasc Disord 2019;19:100-105

6. Yilditz O. How to Safely Implant a Dual-Chamber Pacemaker for Right Ventricular Outflow Tract Pacing in a Patient with Persistent Left Superior Vena Cava: A Step by Step Guide. Acta Cardiol Sin 2019;35:430-432

7. Recupero A, Pugliatti P, Rizzo F et al. Persistent Left-Sided Superior Vena Cava: Integrated Noninvasive Diagnosis. Echocardiography DOI: 10.1111/j.15408175.2007.00509.x 